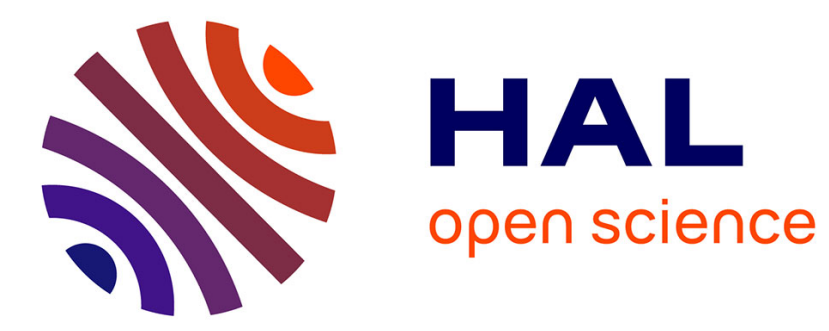

\title{
Positioning principles with a mobile TV system using DVB-SH signals and a Single Frequency Network
}

Paul Thevenon, Olivier Julien, Christophe Macabiau, Damien Serant, Lionel Ries, Stéphane Corazza, Michel Bousquet

\section{- To cite this version:}

Paul Thevenon, Olivier Julien, Christophe Macabiau, Damien Serant, Lionel Ries, et al.. Positioning principles with a mobile TV system using DVB-SH signals and a Single Frequency Network. DPS 2009, 16th International Conference on Digital Signal Processing, Jul 2009, Santorin, Greece. pp 1-8, 10.1109/ICDSP.2009.5201204 . hal-01022205

\section{HAL Id: hal-01022205 \\ https://hal-enac.archives-ouvertes.fr/hal-01022205}

Submitted on 30 Sep 2014

HAL is a multi-disciplinary open access archive for the deposit and dissemination of scientific research documents, whether they are published or not. The documents may come from teaching and research institutions in France or abroad, or from public or private research centers.
L'archive ouverte pluridisciplinaire HAL, est destinée au dépôt et à la diffusion de documents scientifiques de niveau recherche, publiés ou non, émanant des établissements d'enseignement et de recherche français ou étrangers, des laboratoires publics ou privés. 


\title{
POSITIONING PRINCIPLES WITH A MOBILE TV SYSTEM USING DVB-SH SIGNALS AND A SINGLE FREQUENCY NETWORK
}

\author{
Paul Thevenon ${ }^{1,2}$, Olivier Julien ${ }^{3}$, Christophe Macabiau ${ }^{3}$, Damien Serant ${ }^{1,3}$, \\ Lionel Ries ${ }^{4}$, Stéphane Corazza ${ }^{5}$, Michel Bousquet ${ }^{2}$ \\ ${ }^{1}$ TeSA, Toulouse, France \\ ${ }^{2}$ Institut Supérieur de l'Aéronautique et de l'Espace, Toulouse, France \\ ${ }^{3}$ Ecole Nationale de l'Aviation Civile, Toulouse, France \\ ${ }^{4}$ Centre National d'Etudes Spatiales, Toulouse, France \\ ${ }^{5}$ Thalès Alenia Space, Toulouse, France
}

\begin{abstract}
Mobile receiver positioning has received a lot of attention, both in research and standardization bodies, due to regulatory (E-911) or commercial (Location-Based Services) incentives. This paper investigates the possibility of self-positioning a mobile receiver using a mobile TV broadcasting system based on the European DVB-SH standard. This OFDM-based standard offers the interesting possibility of using a Single Frequency Network (SFN) of emitters, meaning that the transmitters are synchronized, which is extremely precious for navigation applications based on Time of Arrival measurements. This paper proposes to base our positioning service on the use of the estimation of the channel done by the receiver, in order to use Time Differences of Arrival techniques. In order to detect several emitters in a SFN, it is proposed to introduce artificial delays between adjacent emitters. The impact of this modification is investigated and some particular DVBSH signal parameters are found to be well-suited to this solution.
\end{abstract}

Index Terms - Mobile TV, positioning, OFDM, channel estimation, single frequency network

\section{INTRODUCTION}

Mobile positioning with wireless networks has been the focus of many research work lately, notably through regulation incentive (USA's E-911, EU's equivalent E112), but also by the potential explosion of Location-Based Services, which promise to generate over $\$ 3.5$ Billion to telecom operators by 2009 [1].

Different telecommunication networks have been investigated, with an important work of standardization at the $3 \mathrm{GPP}$ and ETSI for $2 \mathrm{G}$ and $3 \mathrm{G}$ mobile communication networks [2]. The performances of these positioning solutions vary a lot depending on the positioning method used, which depends on the nature (Cell-ID, Observed Time
Difference of Arrival (O-TDoA) and the location (receiverbased or network-based) of the range measurements and position calculation. The best performance found in literature is a $1 \sigma$ accuracy of $\sim 60 \mathrm{~m}$ using TDoA methods in a $3 \mathrm{G}$ network in urban environment $[3,4]$. Also, since the mobile telecommunication networks are two-way, and have an inherent limitation in their capacity, using them for positioning applications introduces constraints on the rate of positioning requests and the induced capacity robbing required by the transfer of positioning-related information.

Other types of telecommunication networks have been studied, including TV or radio broadcasting network, achieving for example a $1 \sigma$ accuracy of $\sim 20 \mathrm{~m}$ using dedicated ATSC DTV positioning set-up in indoor environment [5].

In this paper, it is proposed to investigate the case of a mobile TV network using the DVB-SH standard. This present case has various challenges, including OFDM fine synchronization and emitters' detection in a Single Frequency Network (SFN), since the motivation for using an SFN is to have the user receiving in a seamless way the signals coming from different transmitters on the same frequency band.

In section 2, the mobile TV broadcast system is presented including the air interface based on DVB-SH and the network topology. In section 3, the targeted positioning principle is described, providing details on the synchronization and channel estimation processes at the origin of the TDoA measurements, and in the last section, we focus particularly on the issue of emitters' detection in a SFN.

\section{PRESENTATION OF A DVB-SH-BASED MOBILE TV BROADCASTING SYSTEM}

\subsection{System architecture and network deployment}

DVB-SH (Digital Video Broadcasting - Satellite to Handheld) is a standard created by ETSI to provide 
television services to mobile users in the S-band [6]. It is derived from the DVB-T (Terrestrial) and DVB-H (Handheld) standards. Its particularity is to provision for the use of a network of emitters composed of one or several geostationary satellites, and a network of terrestrial emitters to be used as gap-fillers in urban environment.

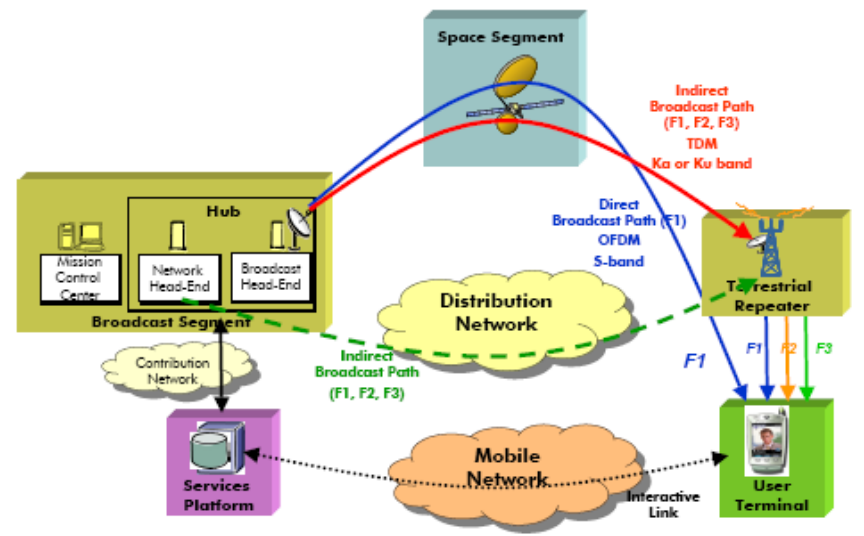

Fig. 1 System architecture of a DVB-SH system [7]

The link budget provided in [10] assesses the System Gain at $150.8 \mathrm{~dB}$ for the terrestrial link (without antenna and SFN diversity). This translates into a $600 \mathrm{~m}$ cell radius using a COST231 Wallfisch Ikegami pathloss model [15], whose parameters are detailed in Table I. Based on this cell radius, a canonical hexagonal terrestrial emitter network deployment is used in our study.

TABLE I

PARAMETERS FOR THE COST231 WALLFISCH IKEGAMI MODEL

\begin{tabular}{|l|c|}
\hline \multicolumn{1}{|c|}{ Parameter } & Value \\
\hline Carrier frequency & $2.2 \mathrm{GHz}$ \\
\hline Building separation & $40 \mathrm{~m}$ \\
\hline Street width & $20 \mathrm{~m}$ \\
\hline Incident wave azimuth & $90^{\circ}$ \\
\hline Roof height & $20 \mathrm{~m}$ \\
\hline Receiver height & $1.5 \mathrm{~m}$ \\
\hline Emitter height & $23 \mathrm{~m}$ \\
\hline
\end{tabular}

The resulting network deployment provides a minimum SNR of $10 \mathrm{~dB}$ (including an $8 \mathrm{~dB}$ shadowing margin) over the whole considered coverage. This seems a reasonable deployment in order to ensure the broadcasting service in multipath and indoor environment.

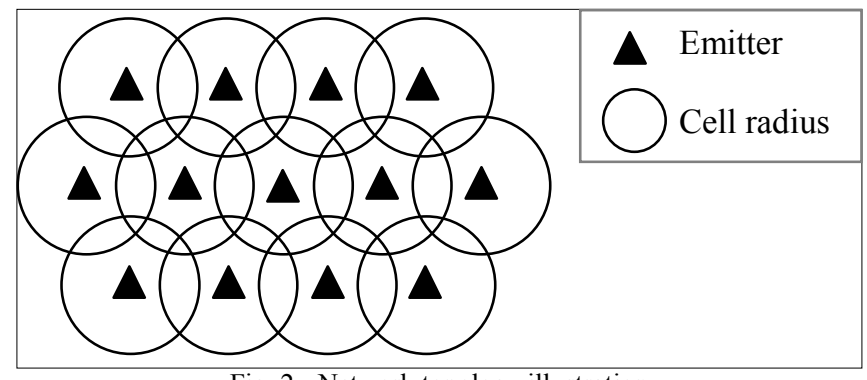

Fig. 2 - Network topology illustration

\subsection{OFDM-based air interface}

The modulation technique used in DVB-SH is based on Orthogonal Frequency Division Multiplexing (OFDM)[6].

OFDM [8] consists in transmitting data symbols over several orthogonal sub-carriers, whose rate is low enough so that the sub-carrier bandwidth is inferior to the channel's coherence bandwidth. Therefore, each sub-carrier will be affected by flat-fading distortion, which can be easily corrected using simple channel estimation techniques.

In order to keep a high spectral efficiency, orthogonal subcarriers are used: adjacent sub-carriers are overlapping, but thanks to the right choice of sub-carrier spacing and pulse shaping, they do not interfere with each other. The transmitted OFDM symbol is then obtained through an inverse FFT. Conversely, a FFT is used at the receiver level to recover the useful data symbols.

Additionally, a guard interval is inserted between successive OFDM symbols in order to avoid Inter-Symbol Interferences (ISI). This guard interval is used to transmit an exact replica of the end of the OFDM symbol, called the Cyclic Prefix (CP). This means that if the FFT operation is done across the $\mathrm{CP}$, it will only result in a phase distortion of the useful symbols, thus no fine synchronization is required.

If a multipath arrives with a delay smaller than the $\mathrm{CP}$ duration, the associated recovered data symbol will only be affected by a complex distortion, which will be corrected by the channel estimation. Therefore, it will not be considered as ISI, but instead it increases the SNR conditions. This illustrates OFDM's robustness against multipath.

An OFDM is defined by 3 main parameters which are the FFT size $\left(N_{F F T}\right)$, the CP length and the transmission bandwidth. DVB-SH considers 36 different sets of signal parameters [6].

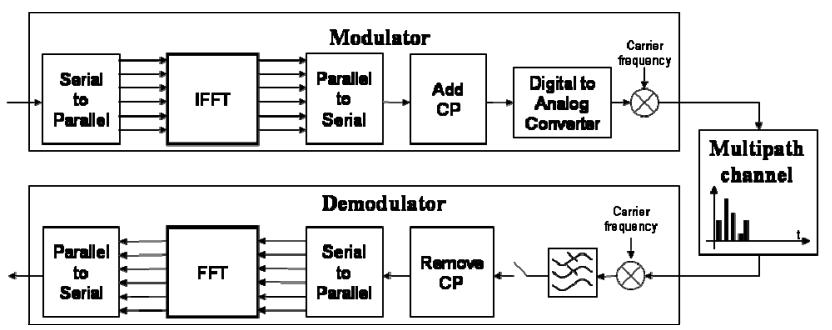

Fig. 3 OFDM transmission block diagram

\subsection{Single Frequency Network}

A SFN is a network where every emitter transmits the same signal on the same carrier frequency in a synchronized way. As seen in Section 2.2 when using OFDM modulation, all the received signals can be added in a coherent manner at the receiver as long as their delay is inferior to the $\mathrm{CP}$ duration. 
This is an extremely interesting feature for telecommunication service, since it improves the service coverage of a network without using more spectrum [9].

For a positioning service, the synchronicity required by a SFN ensures that every emitter has the same time basis, which is essential for accurate positioning based on timing measurements.

\section{TDOA POSITIONING PRINCIPLE AND MEASUREMENTS}

\subsection{TDoA positioning method}

The positioning method retained in this paper is based on the measurement of the Time Difference of Arrival (TDoA) of the signals coming from several emitters. A measurement of TDoA between 2 emitters translates into a hyperbolic location locus [11]. When more than 2 TDoA measurements (3 transmitters) are available, the receiver's horizontal location can be determined at the intersection of the different hyperbolas. TDoA-based positioning requires that the emitters are tightly synchronized, which is the case for emitters working in a SFN without using any extrainfrastructure.

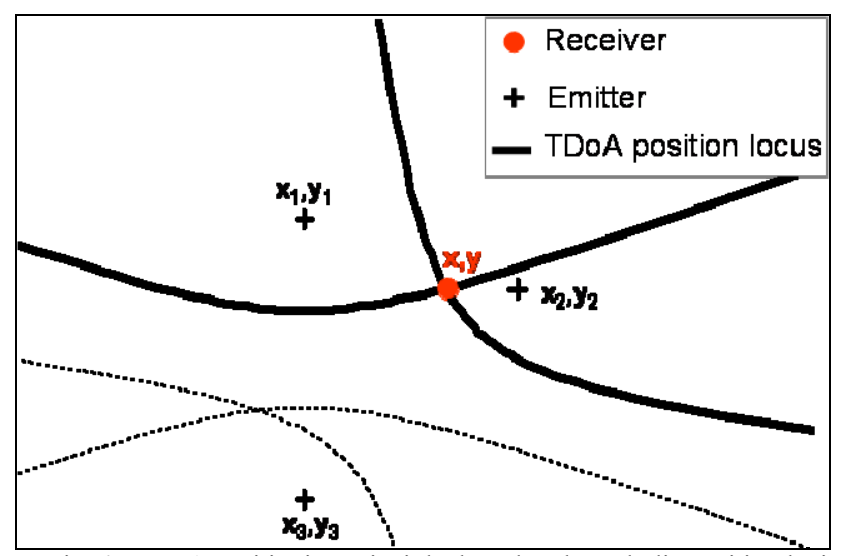

Fig. 4 - TDoA positioning principle, based on hyperbolic position loci

TDoA can be obtained by using the synchronization and the channel estimation measurements, as shown in Fig. 5. The channel estimation provides the estimate of the channel impulse response. By identifying the different taps in the channel impulse response, and associating different groups of taps to one emitter, it will be possible to derive a time of arrival (ToA) coming from one emitter. By subtracting these to a common ToA (e.g. the first emitter's ToA), the receiver can obtain several TDoA measurements.

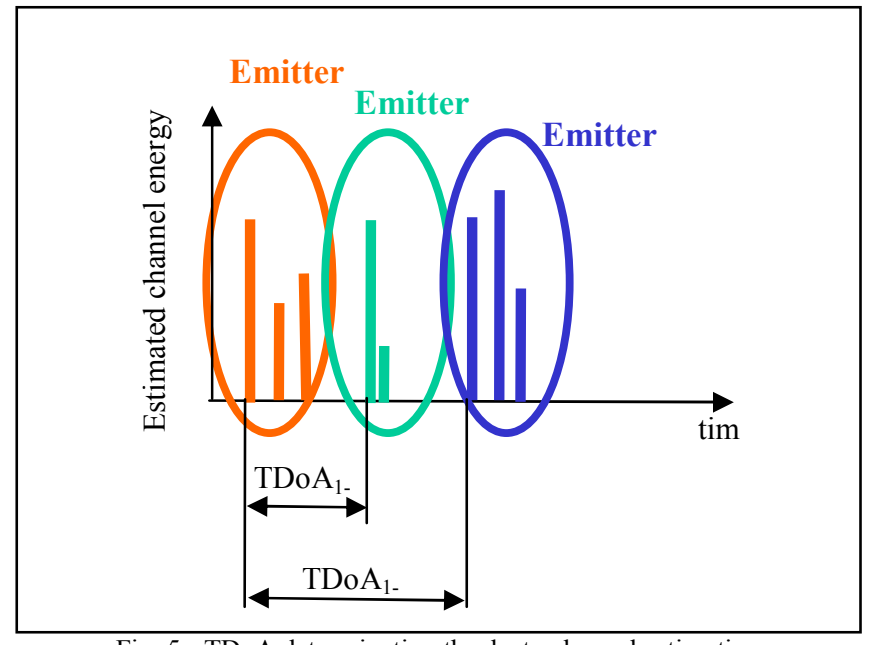

Fig. 5 - TDoA determination thanks to channel estimation

\subsection{OFDM synchronization}

OFDM is a modulation that requires a fine frequency synchronization - in order to minimize Inter-(sub-)Carrier Interference (ICI) - but only a coarse timing synchronization due to the presence of the CP.

Manipulation of expressions given in [12] shows that in presence of a timing error $\Delta t$ and a carrier frequency error $\Delta f$, the estimated data symbol $d_{q}$ present on the $\mathrm{q}^{\text {th }}$ subcarrier can be expressed as a function of all the transmitted data symbols $c_{k}$ by:

$$
d_{q}(\Delta t, \Delta f)=\alpha_{q}(\Delta f) \cdot \sum_{k=0}^{N_{F F T}-1} c_{k} \cdot H_{k} \cdot \beta_{k}(\Delta t) \cdot \gamma_{k, q}(\Delta f)+\mathrm{n}_{\mathrm{q}}
$$

where

- $\quad \alpha_{q}(\Delta f)=e^{j \cdot \pi \cdot \frac{N_{F F T}-1}{N_{F F T}} \cdot\left(N_{F F T} \cdot \Delta f-q\right)}$ is the phase distortion brought by the frequency error,

- $\beta_{k}(\Delta t)=e^{-j \pi \cdot \frac{k \cdot\left(2 \Delta t-N_{F F T}+1\right)}{N_{F F T}}}$ is the phase distortion brought by the timing error

- $\gamma_{k, q}(\Delta f)=\frac{\sin \left(N_{F F T} \cdot \pi \cdot\left(\frac{k-q}{N_{F F T}}+\Delta f\right)\right)}{N_{F F T} \cdot \sin \left(\pi \cdot\left(\frac{k-q}{N_{F F T}}+\Delta f\right)\right)}$

represents the level of ICI due to the frequency error (loss of orthogonality of the sub-carriers);

- $\Delta f$ is the frequency error normalized by the subcarrier spacing;

- $\Delta t$ is the timing error expressed in samples;

- $\quad H_{k}$ is the channel frequency response on the $\mathrm{k}^{\text {th }} \mathrm{sub}-$ carrier;

- $\quad n_{q}$ is a white Gaussian noise. 
It can be seen from Eq. 1 that if $\Delta f=0$, then $\gamma_{k, q}(\Delta f)=0$ all the time except for $k=q$, thus removing completely ICI.

After fine frequency synchronisation and coarse timing synchronisation [12], the frequency offset $\Delta f$ can be considered as null, and the timing offset $\Delta t$ of the order of a few sampling periods.

The output of the OFDM demodulator is then simplified to the following expression:

$$
d_{q}(\Delta t, 0)=c_{q} \cdot H_{q} \cdot e^{-j \cdot 2 \pi \cdot \frac{q \cdot \Delta t}{N_{F F T}}}+n_{q}
$$

The interpretation of this expression is that the $\mathrm{q}^{\text {th }}$ demodulated data symbol is equal to the transmitted data symbol affected by the channel distortion on the corresponding sub-carrier and a phase offset coming from the timing offset. This useful information is further degraded by an additive white Gaussian noise.

This output is used in order to estimate and correct the channel distortion before final decision on the symbols. In our case, we will use the channel estimation process in order to acquire a fine estimation of the signal's delays from different emitters and calculate the corresponding TDoA.

\subsection{OFDM channel estimation using Least Square}

Channel estimation can be done thanks to the use of known data symbols, aka pilot, inserted in the OFDM symbol.

As an example, for the $1 \mathrm{~K}$ signal mode $\left(N_{F F T}=1024\right)$, there are 90 pilot symbols over the 853 symbols transmitted in each OFDM symbol. By comparing the estimated data symbols at the pilot sub-carriers with their known values, it is possible to gather information on the propagation channel.

If we re-write Eq. 2 using matrix expression considering only the pilot sub-carriers, it can be written:

$$
\mathbf{d}=\mathbf{C} \cdot \mathbf{\Delta t} \cdot \mathbf{H}+\mathbf{n}
$$$$
\text { where }
$$

- $\mathbf{d}$ is the vector of the demodulated pilot symbols,

- $\Delta \mathbf{t}=\operatorname{diag}\left(e^{-j 2 \pi \frac{q \Delta t}{N_{F F T}}}\right)$ is a vector representing the impact of the timing offset on the demodulator output, with $q$ taking the values of the pilot subcarrier indexes;

- $\mathbf{n}$ is the noise vector affecting the pilot subcarriers;

- $\mathbf{C}=\operatorname{diag}\left(c_{q}\right)$ is a diagonal matrix containing the transmitted pilot values;

- $\quad \mathbf{H}=\left[\mathbf{H}_{q}\right]^{T}$ is a vector containing the samples of the channel's sampled frequency response on the pilot sub-carriers.
A least-square estimation of the sampled channel's vector is then given by

$$
\hat{\mathbf{H}}_{\Delta \mathbf{t}}=\Delta \mathbf{t} \cdot \mathbf{H}=\mathbf{C}^{-1} \cdot \mathbf{d} \text {. }
$$

This estimation comprises both the effect of the frequencyselective channel and of the remaining timing offset.

\subsection{OFDM channel estimation refinement}

Following the previous technique, the channel is estimated only on the pilot sub-carriers. In order to have the channel frequency response on every sub-carrier, it is necessary to interpolate it on the data sub-carriers. For this, a simple linear interpolation can be used as shown in Fig. 6 .

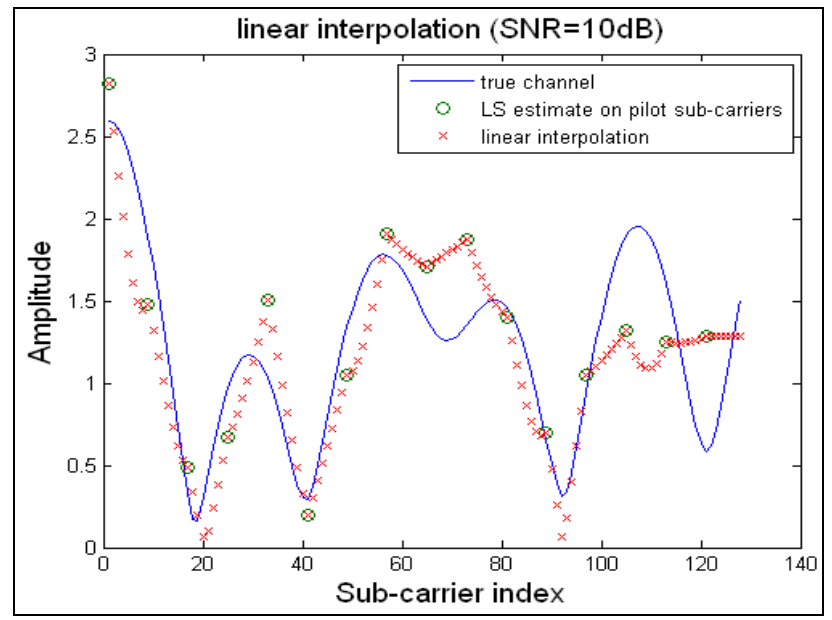

Fig. 6 - Estimated channel on pilot sub-carriers and linear interpolation

Furthermore, the evolution of the multipath channel's response can be assumed to follow a Jakes' spectrum [13]. This induces that the evolution of the channel's response is concentrated below the frequency Doppler

$f_{D}=v / \lambda_{c}$

where

- $\quad v$ is the relative speed between the receiver and the emitter;

- $\quad \lambda_{c}$ is the wavelength of the carrier frequency.

In order to further decrease the noise component, it is therefore interesting to filter the estimated channel frequency response obtained using successive OFDM symbols by a low-pass filter with a cut-off frequency slightly above the Doppler frequency. This smoothes the estimate of the channel estimated response, as illustrated in Fig. 7. 


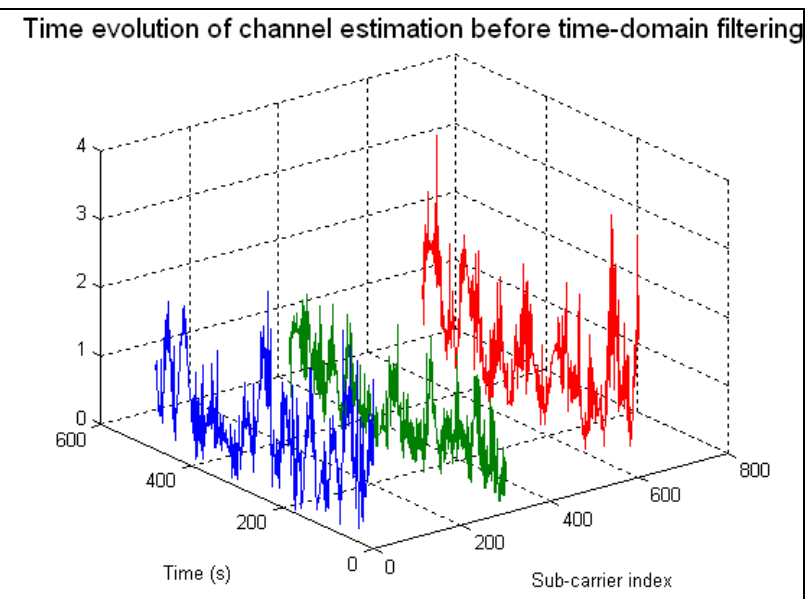

Time evolution of channel estimation after time-domain filtering

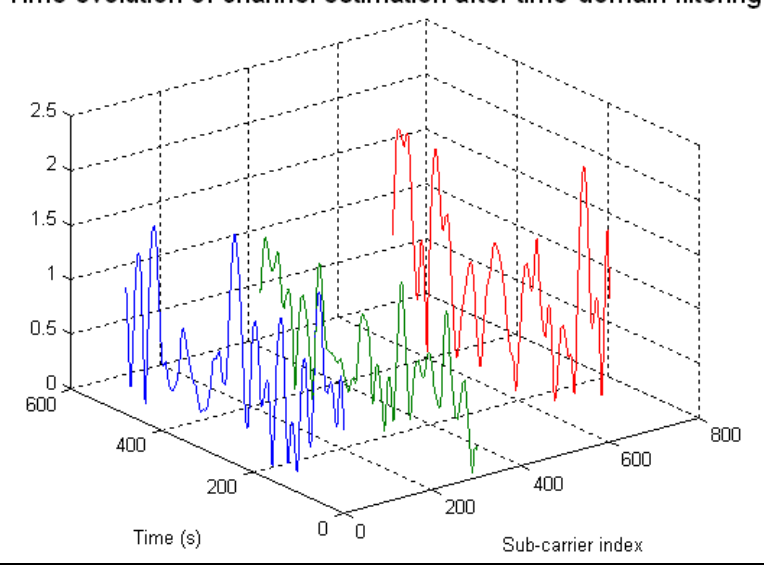

Fig. 7 - Impact of low-pass filtering on the channel frequency response estimate corresponding to 3 sub-carriers

These two techniques greatly improve the accuracy of the channel estimation at low SNR. The propagation channel model [13] coming from a single emitter was estimated with these techniques, and the Mean Square Error between the estimated channel and the real one is depicted in Fig. 8.

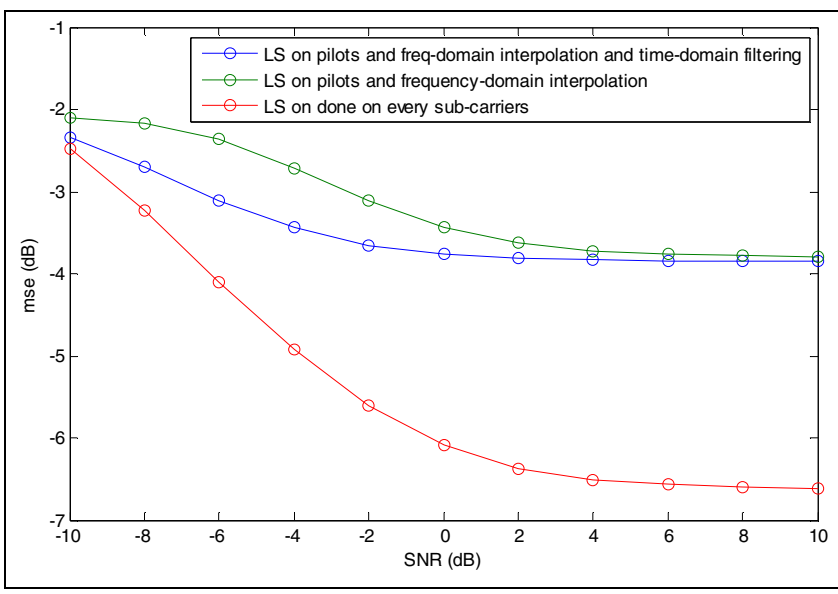

Fig. 8 - Accuracy of the channel estimation using different techniques

\section{EMITTERS' DETECTION IN A SFN}

\subsection{SFN Channel modeling}

This section deals with the propagation channel applied to a signal transmitted by a SFN with the network topology illustrated in Fig. 2.

In order to model it, we apply a realization of the channel model [13] to each emitter, and then correct it with a delay and attenuation depending on the distance between the emitter and the receiver.

The applied attenuation follows the COST 231 Wallfisch Ikegami pathloss model [14] in urban environment with the parameters described in Table I. Shadowing was not simulated.

The resulting channel impulse response realization averaged over 1s for a mobile moving at $50 \mathrm{~km} / \mathrm{h}$ is shown in Fig. 9.

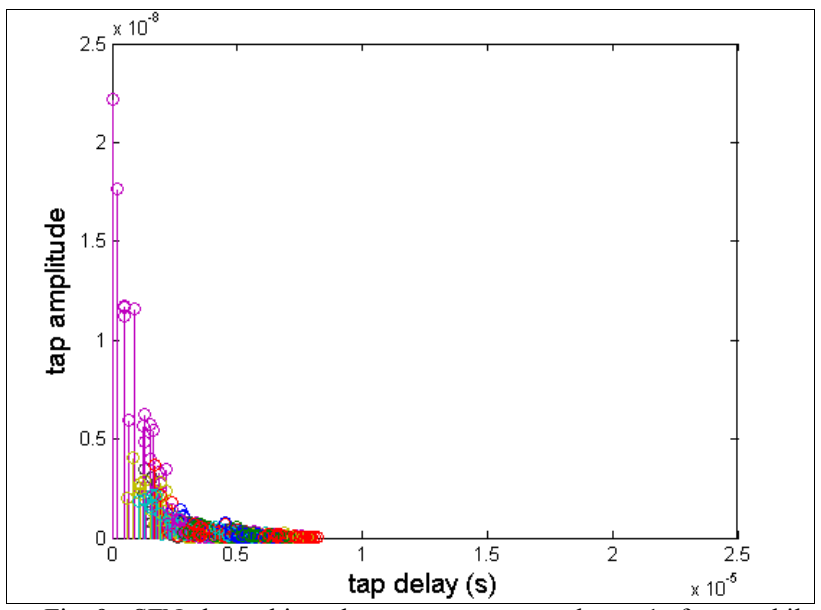

Fig. 9 - SFN channel impulse response averaged over 1s for a mobile receiver speed of $50 \mathrm{~km} / \mathrm{h}$

\subsection{Emitter detection issue in a SFN}

In order to calculate the TDoA from the signal arriving from different emitters, it is necessary that the signals coming from one emitter are distinguishable from those coming from another.

Looking at Fig. 9, this seems impossible since exactly the same signal is sent by each transmitter. To distinguish two emitters, the associated TDoA cannot be below the longest delay of the last significant multipaths, also known as the channel's delay spread.

Considering [13], the typical channel delay spread for an urban environment is $2 \mu \mathrm{s}$, which translates into a distance of $600 \mathrm{~m}$. Therefore, if the receiver is in a zone where the difference of distances to 2 emitters is less than $600 \mathrm{~m}$, it will not be able to detect the presence of 2 emitters. We call these interference zones iso-delay zones.

In the chosen network deployment, the cell radius is $600 \mathrm{~m}$, which means that the whole service coverage is an iso-delay zone. 


\subsection{Introduction of artificial delay}

In order to solve this problem, it is proposed to introduce an artificial delay to the signal's emission between adjacent emitters.

From the observation of section 4.2, this delay should be superior to the channel delay spread.

To avoid ISI, another constraint is that this delay should be less than the CP length minus the channel delay spread.

The CP length for the different modes of DVB-SH signals are summarized in Table II.

TABLE II

CP LENGTH OF DVB-SH SIGNALS

\begin{tabular}{|c|c|c|c|c|c|}
\hline \multirow{2}{*}{ Mode } & \multirow{2}{*}{ Bandwidth } & \multicolumn{5}{|c|}{ CP Duration $(\boldsymbol{\mu s})$} \\
\cline { 3 - 6 } & & $\mathbf{1 / 4}$ & $\mathbf{1 / 8}$ & $\mathbf{1 / 1 6}$ & $\mathbf{1 / 3 2}$ \\
\hline $1 \mathrm{~K}$ & $5 \mathrm{MHz}$ & 44.8 & 22.4 & 11.2 & 5.6 \\
\hline $2 \mathrm{~K}$ & $5 \mathrm{MHz}$ & 89.6 & 44.8 & 22.4 & 11.2 \\
\hline $4 \mathrm{~K}$ & $5 \mathrm{MHz}$ & 179.2 & 89.6 & 44.8 & 22.4 \\
\hline $8 \mathrm{~K}$ & $5 \mathrm{MHz}$ & 358.4 & 179.2 & 89.6 & 44.8 \\
\hline $1 \mathrm{~K}$ & $1.7 \mathrm{MHz}$ & 140 & 70 & 35 & 17.5 \\
\hline $1 \mathrm{~K}$ & $8 \mathrm{MHz}$ & 28 & 14 & 7 & 3.5 \\
\hline $2 \mathrm{~K}$ & $8 \mathrm{MHz}$ & 56 & 28 & 14 & 7 \\
\hline $4 \mathrm{~K}$ & $8 \mathrm{MHz}$ & 112 & 56 & 28 & 14 \\
\hline $8 \mathrm{~K}$ & $8 \mathrm{MHz}$ & 224 & 112 & 56 & 28 \\
\hline
\end{tabular}

These loose constraints allows planning for several artificial delay values for different emitters, and also to re-use the delay value for remote emitters, similarly to the frequency re-use scheme done in GSM to avoid interferences. Introducing an artificial delay between two adjacent emitters does not remove iso-delay zones, but rather moves them away. However, it is possible to move them far away enough so that a receiver in the modified iso-delay zone will not be in the service zone of one of the two emitters.

The choice of the reuse factor is a trade-off between the reuse distance (how far 2 emitters with the same delay will be) and the maximum artificial delay introduced in the SFN. We have chosen a 7-color pattern, with a $2.5 \mu$ s artificial delay step, meaning that the maximum introduced artificial delay is $15 \mu \mathrm{s}$. Therefore, we should use a DVB-SH signal with a CP length value over this maximum artificial delay plus the channel's delay spread and a margin due to propagation delay. This leads to recommend DVB-SH signals with CP length over $20 \mu$ s.

By applying a 7-color pattern, the iso-delay zones in our network are much reduced, as shown in Fig. 10. Without this, the whole service area would be considered as an isodelay zone and TDoA estimation would not be possible.

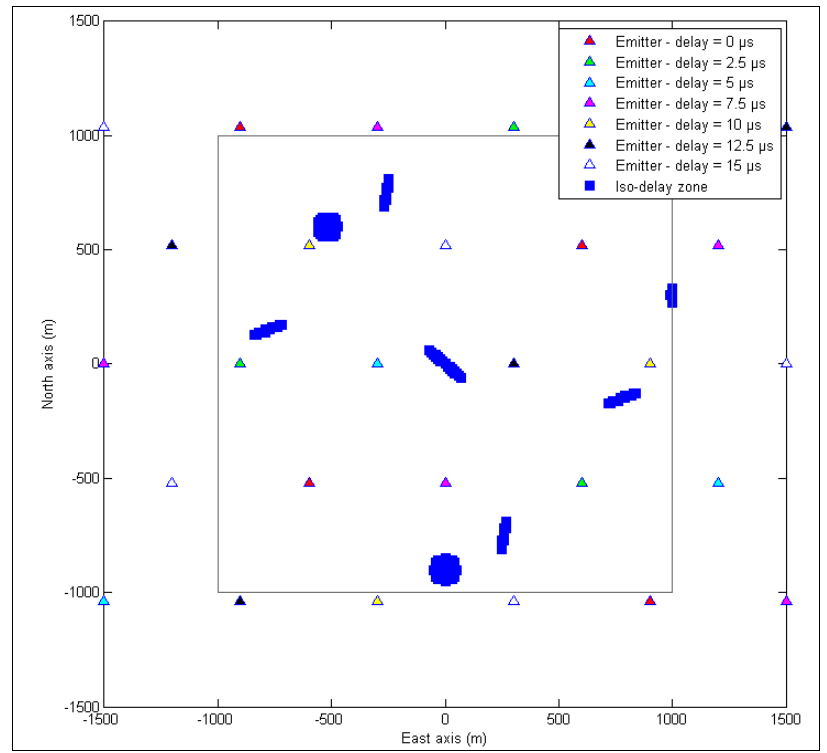

Fig. 10 - Iso-delay zone for a 7-color delay re-use pattern. The emitter's cell radius is limited to $800 \mathrm{~m}$, while the emitter separation is $600 \mathrm{~m}$.

The modified channel impulse response averaged over $1 \mathrm{~s}$ is shown in Fig. 11. This channel impulse response paves the way towards detecting the presence of several emitters in a SFN.

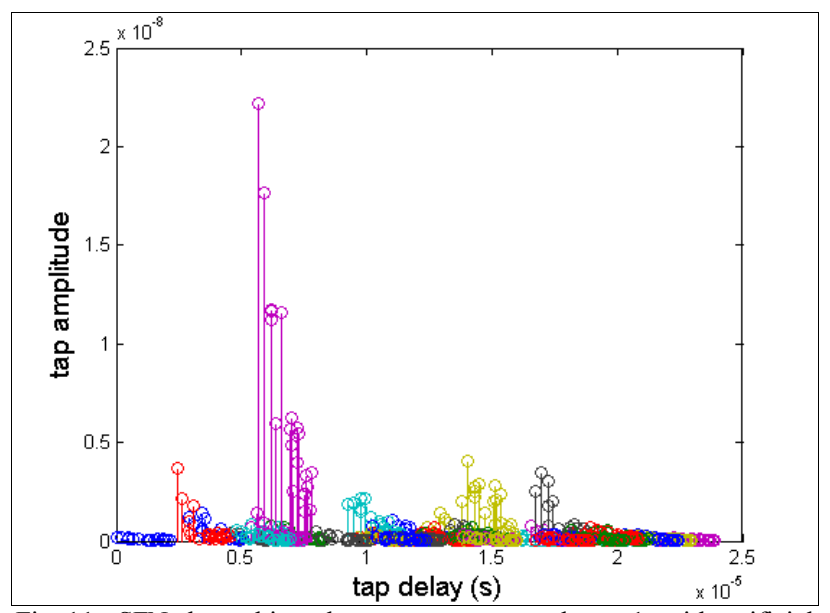

Fig. 11 - SFN channel impulse response averaged over 1s with artificial delay (same channel as Fig. 9)

\subsection{Maximum observable delay}

A channel with larger delays (of tens of $\mu$ s instead of $\sim 2 \mu \mathrm{s}$ ) has a narrower coherence bandwidth, and thus requires an increased density of pilot symbols. Indeed, a delayed multipath transposes in the frequency domain into a complex exponential whose period is proportional to the delay value. Due to the Shannon's sampling theorem, the maximum observable oscillation rate of the channel frequency response is directly related to the spectral density of pilots, by the following formula: 
$\tau_{\max }=\frac{1}{2} \frac{N_{\text {pilots }}}{B}$

where $B$ is the transmission useful bandwidth;

$N_{\text {pilots }}$ is the number of pilots in this mode;

This resolution is the one obtained by the estimation of a single OFDM symbol. Since some pilot symbols are placed at different position positions in successive OFDM symbols, the resulting pilot density could be larger when processing several OFDM symbols at the same time.

Table III summarizes the different accuracies expectable from the different signal profiles of DVB-SH.

TABLE III

MAXIMUM OBSERVABLE DELAY OF DVB-SH MODES

\begin{tabular}{|c|c|c|c|}
\hline $\begin{array}{c}\text { Mode \& } \\
\text { Bandwidth }\end{array}$ & $\begin{array}{c}\text { Number } \\
\text { of pilot } \\
\text { symbols }\end{array}$ & $\begin{array}{c}\text { Useful } \\
\text { bandwidth } \\
\text { (MHz) }\end{array}$ & $\begin{array}{c}\text { Maximum } \\
\text { observable delay } \\
(\boldsymbol{\mu s )})\end{array}$ \\
\hline $8 \mathrm{~K}-5 \mathrm{MHz}$ & 701 & 4.161 & 84.2 \\
\hline $8 \mathrm{~K}-8 \mathrm{MHz}$ & 701 & 6.657 & 52.6 \\
\hline $4 \mathrm{~K}-5 \mathrm{MHz}$ & 351 & 4.161 & 42.2 \\
\hline $1 \mathrm{~K}-1.7 \mathrm{MHz}$ & 90 & 1.416 & 31.8 \\
\hline $4 \mathrm{~K}-8 \mathrm{MHz}$ & 351 & 6.657 & 26.4 \\
\hline $2 \mathrm{~K}-5 \mathrm{MHz}$ & 176 & 4.163 & 21.1 \\
\hline $2 \mathrm{~K}-8 \mathrm{MHz}$ & 176 & 6.660 & 13.2 \\
\hline $1 \mathrm{~K}-5 \mathrm{MHz}$ & 90 & 4.165 & 10.8 \\
\hline $1 \mathrm{~K}-8 \mathrm{MHz}$ & 90 & 6.664 & 6.8 \\
\hline
\end{tabular}

In Fig. 13, the different DVB-SH signal parameters are classified according to the $\mathrm{CP}$ duration (Table II) and to the maximum observable delay permitted by the pilot density (Table III).

Different zones can be defined, according to their relevance to our proposed solution. They are shown in Fig. 12.

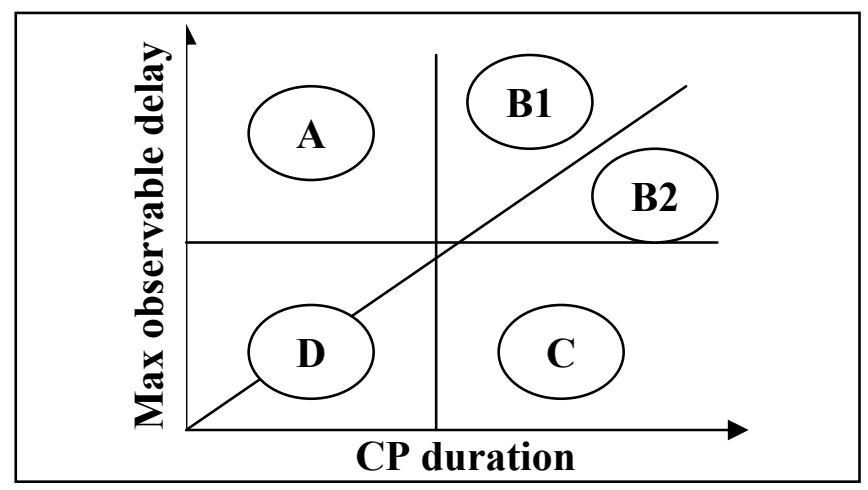

Fig. 12 - Categories of DVB-SH parameters

Zone A and D correspond to the DVB-SH signal parameters where the CP duration is less than $20 \mu$ s. In this case, a high-order delay reuse scheme cannot be implemented, which will lead to the impossibility to reduce the iso-delay zones.
Zone $\mathrm{C}$ and $\mathrm{D}$ correspond to the DVB-SH signal parameters where the maximum observable delay is inferior to $20 \mu \mathrm{s}$. In this case, even if high delays can be introduced in the emitters' network (as in zone $\mathrm{C}$ ), the receiver will not be directly able to observe the longest delay echos, due to the insufficient density of pilots. Still, long-delay emitters may be detectable thanks to advanced signal processing techniques (e.g. decision-directed channel estimation) or by the introduction of additional pilots in some OFDM symbols.

Zone B is the zone of most interest, since it allows for the introduction of high delays, most of which will be observable. It is further divided in zone B1, where the every introduced delays will be observable, and in zone B2, where the longest delays will not be observable.

\subsection{Introduction of artificial delay: Pros, cons and further work}

We have shown that the introduction of an artificial delay at the emitters allows a receiver to detect several emitters in an SFN based on the estimated channel impulse response realised thanks to the presence of pilots.

The advantage of this method is that as long as the signals arrive inside the $\mathrm{CP}$ duration and the channel can be estimated, the signal processing required for data demodulation should not be affected by the presence of these artificial echos. This is possible thanks to the OFDM's robustness against multipaths.

Another advantage is that the DVB-SH standard has already provisioned for the introduction of artificial delay. This is in fact a legacy feature which was introduced in the DVB-T (fixed TV broadcasting standard) in order to accommodate the very long delay of signals arriving from emitters with different radiating power (macro-cells vs micro-cells).

Therefore, this modification should be realizable with only minor modifications to the planned DVB-SH systems.

However, the introduction of artificial delay also has drawbacks. First, in order to optimize the iso-delay zone reduction, it may be necessary to have a high delay re-use factor, which may impose using a long Cyclic Prefix. Using longer than required $\mathrm{CP}$ is detrimental to the system capacity, since it means that a larger duration of the transmission will not be dedicated to data transmission.

Secondly, the artificial increase of the propagation delays of the echos in the SFN channel reduces the zone where only a coarse timing synchronization is sufficient. This "safe" zone is the time interval between the last considered echo of the channel impulse response and the end of the CP. If the remaining time offset after coarse synchronization is in this safe zone, then it will be corrected through channel estimation. If it falls out of this safe zone, then ISI will appear and degrade the transmission efficiency. Therefore obtaining more accurate timing synchronization processes may be required in order to ensure sufficiently good 
synchronization before passing the samples in the OFDM demodulator and channel estimation process.

Finally, the introduction of long artificial delays requires a high density of pilots, which is available only in particular DVB-SH signal parameters.

This has led us to the conclusion that some DVB-SH signal parameters are better suited to our positioning principle. In order to benefit from a positioning service, these additional constraints should be taken into account in the trade-offs studies when designing a DVB-SH-based systems.

\section{CONCLUSION}

The principle of a new positioning service based on the use of DVB-SH systems is proposed. Indeed, with the possibility to use SFN, DVB-SH systems are well suited to TDoA-based positioning methods. TDoA are measured thanks to synchronization and channel estimation algorithms. The problem of iso-delay zones - which would prevent the detection of the arrival of the signal coming from 2 different emitters in the same time window - is solved by the introduction of artificial delays between the emission of the signal of neighboring SFN emitters. With the right choice of DVB-SH signal parameters, this solution would require only minor modifications in the system deployment and the receiver's algorithms, but trade-off studies are still required in order to determine the optimal sharing of resources between the broadcasting and the positioning service.

\section{REFERENCES}

[1] Alcatel Location Based Solutions - White Paper. 2006. Available at http://www.liaison-project.eu/download/2006-11-15\%20LBS.pdf
[2] ETSI TS 123271 - Digital cellular telecommunication system (Phase 2+); Universal Mobile Telecommunications System (UMTS); LTE; Function stage 2 description of Location Services (LCS) (3GPP TS 23.271). 2009.

[3] Ludden B, Lopes L. Cellular based location technologies for UMTS a comparison between IPDL and TA-IPDL. In: Vehicular Technology Conference Proceedings, 2000. VTC 2000-Spring Tokyo. 2000 IEEE 51st. vol. 2; 2000. p. 1348-1353 vol.2.

[4] Porcino D. Performance of a OTDOA-IPDL positioning receiver for 3GPP-FDD mode. In: 3G Mobile Communication Technologies, 2001. Second International Conference on (Conf. Publ. No. 477); 2001. p. 221-225.

[5] Rabinowitz M, Spilker JJ. A new positioning system using television synchronization signals. In: IEEE Transactions on Broadcasting, Vol. 51, No. 1. (2005), pp. 51-6; 2005.

[6] ETSI EN 302583 - Digital Video Broadcasting (DVB); Framing Structure, channel coding and modulation for Satellite Services to Handheld devices (SH) below 3 GHz. 2008.

[7] Laine P. Alcatel-Lucent's proposal for unlimited mobile TV: a hybrid satellite/terrestrial broadcast network based on DVB-SH; 2007.

[8] Bingham JAC. Multicarrier modulation for data transmission: an idea whose time has come. Communications Magazine, IEEE. 1990; 28(5):5-14.

[9] Fernández J, Capdevila J, García R, Cabanillas S, Mata S, Mansilla A, et al. Single Frequency Networks for Digital Video Broadcasting; 1999. p. 120-142.

[10] Chuberre N, Bodevin F, Courseille O, Duval R, Dussauby E, Selier C. Unlimited Mobile TV. In: ASMS 2006

[11] Gustafsson F, Gunnarsson F. Mobile positioning using wireless networks: possibilities and fundamental limitations based on available wireless network measurements. Signal Processing Magazine, IEEE. 2005;22(4):41-53.

[12] Morelli M, Kuo CCJ, Pun MO. Synchronization Techniques for Orthogonal Frequency Division Multiple Access (OFDMA): A Tutorial Review. Proceedings of the IEEE. 2007;95(7):1394-1427.

[13] ETSI TR 125943 v6.0.0 - Universal Mobile Telecommunications System (UMTS); Deployment aspects (3GPP TR 25.943). 2004.

[14] Digital Mobile Radio Towards Future Generation Systems - COST 231 Final Report. 1993

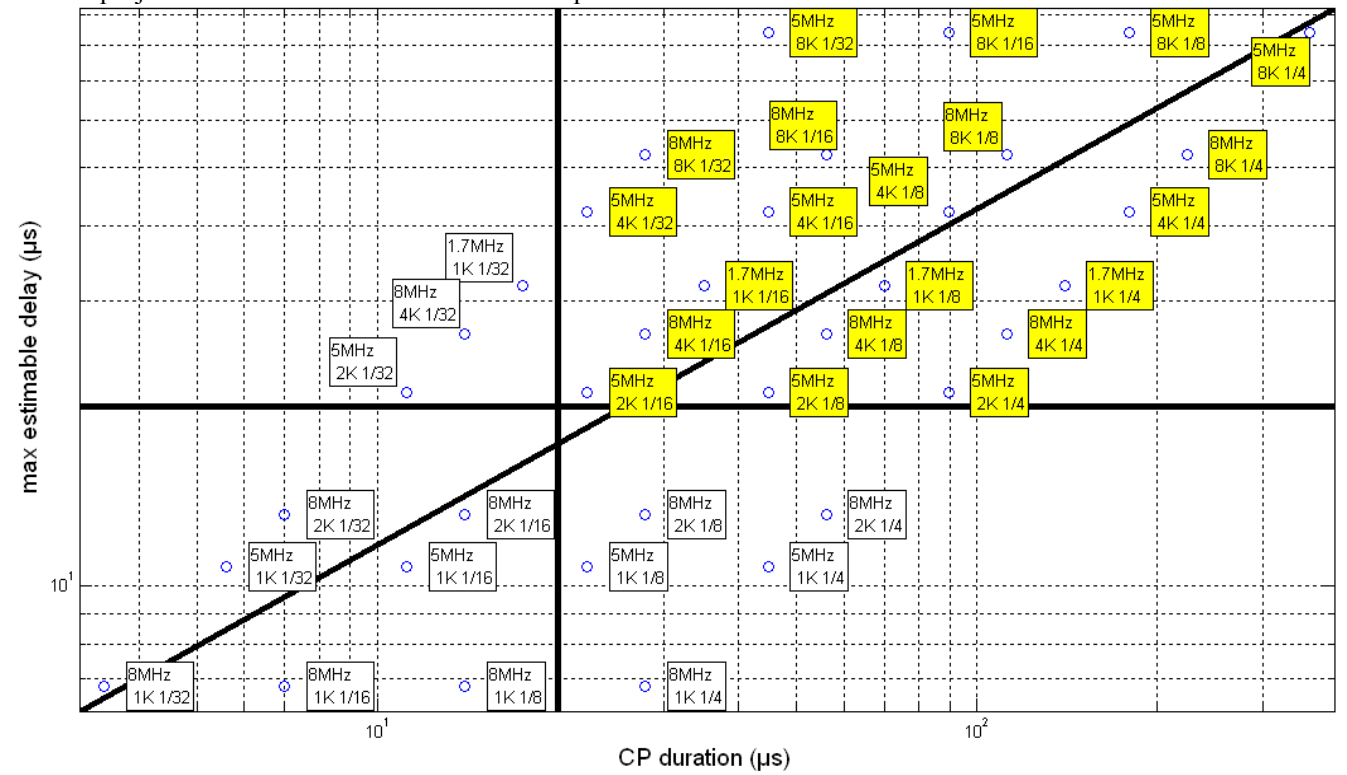

Fig. 13 - Classification of DVB-SH signal parameters according to CP duration and maximum observable delay. The highlighted modes are recommended by the authors. 